\title{
Quantum vacuum fluctuations and dark energy
}

\author{
Emilio Santos \\ Departamento de Física. Universidad de Cantabria. \\ Santander. 39005 Spain
}

September, 21, 2009

\begin{abstract}
It is shown that the curvature of space-time induced by vacuum fluctuations of quantum fields should be proportional to the square of Newton constant $G$. This offers a possible explanation for the success of the formula $\rho \sim G m^{6} c^{2} h^{-4}, \rho$ being the dark energy density and $m$ a typical mass of elementary particles.
\end{abstract}

PACS: 04.60.-m; 98.80.Hw

Keywords: Dark energy; Vacuum fluctuations; Quantum gravity.

The observed accelerated expansion of the universe [1] is currently assumed to derive from a positive mass density and a negative pressure, constant throughout space and time, which is known as "dark energy". The mass density, $\rho_{D E}$, and the presure, $p_{D E}$, are [2]

$$
\rho_{D E} \simeq-p_{D E} \simeq 10^{-26} \mathrm{~kg} / \mathrm{m}^{3} .
$$

Many proposals have been made for the origin of dark energy (for a review see [3] ). The most popular is to identify it with the cosmological constant $\Lambda$ introduced by Einstein in 1917 or, what is equivalent in practice, to assume that it derives from the quantum vacuum[4]. Indeed the equality $\rho_{D E}=$ $-p_{D E}$ is appropriate for the vacuum (in Minkowski space, or when the spacetime curvature is small) because it is invariant under Lorentz transformations.

A problem appears however when one attempts to estimate the value of $\rho_{D E}$. In particular if the dark energy is due to the interplay between quantum 
mechanics and gravity, it should be either strictly zero or of order Planck's density, that is

$$
\rho_{D E} \sim \frac{c^{5}}{G^{2} \hbar} \simeq 10^{97} \mathrm{~kg} / \mathrm{m}^{3} .
$$

This is about 123 orders of magnitude larger than eq.(11) [5]. Some explanations have been proposed for the huge value of the ratio between the Planck density, eq.(2), and the dark energy density, eq.(11). For instance Novello 6] starts proving that, if $\Lambda$ is a fundamental parameter, to be put on the left side of the Einstein equation (eq.(8) below), the space-time in the absence of matter is de-Sitter and the mass of the graviton is not zero. Then the ratio between eq.(2) and eq.(11) may correspond to the number of gravitons in the visible universe. On the other hand if $\Lambda$ has to do with the matter content of the vacuum, it should appear on the right side of the Einstein equation. In this case $\rho_{D E}$ should be related to other fundamental constants. Support to the latter assumption is the fact that a fairly good fit to eq.(1) may be obtained from a specific combination of the fundamental constants $G, \hbar$ and $c$ plus a mass parameter $m$ of the order of a typical mass of fundamental particles, that is [7]

$$
\rho_{D E} \sim G \frac{m^{6} c^{2}}{\hbar^{4}}
$$

Actually the observed value, eq.(11), is obtained if the mass $m$ is

$$
m \sim 7.6 \times 10^{-29} \mathrm{~kg}
$$

which is about $1 / 20$ times the proton mass or about 80 times the electron mass.

I believe that dark energy is indeed associated to the matter content of the quantum vacuum and the purpose of the present paper is to provide a possible explanation for the estimate eq.(3) . Furthermore I propose that dark energy derives from the quantum vacuum fluctuations, an assumption considered recently by Padmanabhan[8], but my approach here is different. It rests upon the interplay between quantum mechanics and general relativity. Thus we shall work within quantized gravity. If we choose an appropriate coordinate system in the universe, with coordinates

$$
x \equiv\left\{x_{1}, x_{2}, x_{3}, x_{4}\right\},
$$

our aim is to relate the quantum state, $|\Phi\rangle$, and the stress-energy tensor operator, $\widehat{T}_{\mu}^{\nu}(x)$, of quantum fields with the space-time structure. That 
structure is given by the metric tensor operator, $\widehat{g}_{\mu \nu}$. The study of the quantum fields in curved space-times and the gravitational back reaction of the fields is a difficult subject [9]. However for the purposes of the present paper it is enough to assume that the expectation value of the stress-energy tensor may be written in the form

$$
\left\langle\Phi\left|\widehat{T}_{\mu}^{\nu}\right| \Phi\right\rangle=\left(T^{m a t}\right)_{\mu}^{\nu}+\left(T^{v a c}\right)_{\mu}^{\nu}
$$

where $\left(T^{m a t}\right)_{\mu}^{\nu}$ is the stress-energy tensor associated to matter, baryonic and possibly dark, plus radiation, and $\left(T^{v a c}\right)_{\mu}^{\nu}$ the tensor due to the vacuum. Both terms may be treated as classical. The latter contribution might be relevant when the space-time metric departs substantially from Minkowski's. This may be the case in the early universe (so explaining "inflation") or near galaxies (so explaining "dark matter"). Both these possibilities have been explored recently using $f(R)$-gravity [10], [11] .

It is convenient to define a stress-energy tensor operator, $\left(\widehat{T}^{\text {fluct }}\right)_{\mu}^{\nu}$, such that eq.(5) may be rewritten

$$
\left\langle v a c\left|\left(\widehat{T}^{f l u c t}\right)_{\mu}^{\nu}\right| v a c\right\rangle=0,\left(\widehat{T}^{\text {fluct }}\right)_{\mu}^{\nu}=\widehat{T}_{\mu}^{\nu}-\widehat{I}\left(T^{v a c}\right)_{\mu}^{\nu},
$$

where $\widehat{I}$ is the identity operator. The existence of vacuum fluctuations means that, although the expectation of $\left(\widehat{T}^{\text {fluct }}\right)_{\mu}^{\nu}$ is zero, there are correlated fluctuations, that is

$$
\left\langle v a c\left|\left(\widehat{T}^{\text {fluct }}(x)\right)_{\mu}^{\nu} \times\left(\widehat{T}^{\text {fluct }}(y)\right)_{\lambda}^{\sigma}\right| v a c\right\rangle \neq 0 \text { in general. }
$$

For the sake of simplicity I shall ignore the matter terms on the right hand side of eq.(5) and write $\widehat{T}_{\mu}^{\nu}$ for $\left(\widehat{T}^{\text {fluct }}\right)_{\mu}^{\nu}$ in the following.

After that I return to the problem of the possible explanation for the agreement of eq.(3) with observations, eq.(1). I shall start recalling a well known prediction of quantum mechanics, namely that correlations between quantum fluctuations may produce observable effects. An illustrative example is the (van der Waals) interaction between two molecules at a distance, $d$, much bigger than the typical size of a molecule. If both molecules possess a permanent electric dipole moment, then at low enough temperature they are oriented so that the molecules attract each other. In fact there is a dipoledipole (negative) interaction energy which scales as $d^{-3}$. Now let us consider two neutral molecules which do not possess permanent dipole moment. In 
this case classical physics predicts that there is no electrostatic interaction between them. Quantum theory however predicts an interaction due to the fact that quantum fluctuations give rise to instantaneous dipole moments in both molecules, which are correlated so that the energy is a minimum. This gives rise to an interaction which scales precisely as the square of the coupling parameter above mentioned, that is $\left(d^{-3}\right)^{2}=d^{-6}$. Indeed it is well known that the interaction energy between nonpolar molecules decreases with the six power of the distance (when retardation effects are negligible).

The general behaviour may be understood via perturbation theory. To first order an average of the quantum fluctuations appears, which is zero (this is similar to eq.([6).) To second order however the perturbation involves the product of two correlated fluctuations, which is not zero (this is similar to eq.(17) and it gives the interaction to lowest order. The square of the quantum fluctuations involves the coupling constant squared.

I propose that a similar phenomenon should appear in gravity. Classical general relativity predicts that any stress-energy, $T_{\mu \nu}$, produces curvature of the space-time measured by the Ricci tensor, $R_{\mu}^{\nu}$, the scaling parameter being Newton constant, $G$. Indeed this is the essential content of Einstein equation

$$
R_{\mu}^{\nu}-\frac{1}{2} g_{\mu}^{\nu} R=8 \pi c^{-2} G T_{\mu}^{\nu}
$$

The point is that, if there existed a classical non-fluctuating stress-energy, the curvature would scale as $G$. However if there is no classical stress-energy, in particular if the vacuum expectation of the stress-energy tensor is zero, then there is no curvature to order $G$, but there will be an induced curvature to order $G^{2}$.

Gravity theory is more involved than molecular theory because the equations of the former are non-linear. In addition there is not yet a satisfactory quantum gravity theory. Consequently a rigorous derivation of the curvature due to vacuum fluctuations is not possible at this moment. Nevertheless we may conjecture that the curvature due to vacuum fluctuations should be $G^{2}$ times some weighted average of the correlations of the quantum vacuum stress-energy tensor at two different space-time points. I stress that here I consider fluctuations of all quantum fields (e.g. electromagnetic radiation field, electron-positron field, etc.) and try to compute the gravitational effects of these fluctuations. Now the vacuum expectation, $\left\langle v a c\left|\widehat{g}_{\mu \nu}\right| v a c\right\rangle$, of the metric tensor, $\widehat{g}_{\mu \nu}$, may be taken as a classical metric, which should be used to calculate distances and time intervals. Indeed the accelerated ex- 
pansion of the universe has been measured by relating distances and times of supernavae events in distant galaxies 1 . From the vacuum expectation of the metric tensor we may derive the Ricci tensor, $R_{\mu}^{\nu}$, which measures the curvature induced by the quantum vacuum fluctuations. Thus I may write schematically

$$
R_{\mu}^{\nu} \sim G^{2} \times\langle q \text { uantum correlation }\rangle,
$$

where 〈quantum correlation〉 stands for some appropriate average of the quantum correlations eq.(7) .

As said above deriving a quantitative expression rather than eq.(9) would require a quantum gravity theory not yet available. (In order to illustrate the main features of the derivation I shall work below a toy model which is easily soluble). However, a rough estimate of the term 〈quantum correlation may be obtained by dimensional analysis. In fact, $R_{\mu}^{\nu}$ has dimensions of inverse length squared. Thus the term 〈quantum correlation $\rangle$ should have dimensions of $(G L)^{-2}$, that is $(\text { mass })^{2}(\text { time })^{4}(\text { length })^{-8}$. This should be obtained from typical parameters of quantum theory that is the speed of light, $c$, Planck's constant, $\hbar$, and some typical mass of elementary particles, $m$. We obtain

$$
\langle\text { quantum correlation }\rangle \sim \frac{m^{6}}{\hbar^{4}} .
$$

Here I have excluded Newton constant, $G$, because at very low curvature, as in the universe at the present epoch, we should work at the lowest possible order in $G$, that is just second order as in eq.(9) .

Now we may ask: Is there a classical density, $\rho_{D E}$, which could mimic the effect ot the vacuum fluctuations as given by eqs.(9) and (10)? A comparison with Einstein eq.(8) gives an affirmative answer provided that

$$
c^{-2} G \rho_{D E} \sim G^{2} \frac{m^{6}}{\hbar^{4}} .
$$

This leads precisely to eq.(3). We conclude that the quantum vacuum fluctuations should give rise to a curvature of space-time of the order attributed to the "dark energy". Whether there are additional contributions to dark energy will not be studied in the present paper.

In the following I shall work a simple toy model within quantized gravity for illustrative pursposes. As quantum gravity theory is not yet available, I shall introduce a few plausible assumptions. I consider that the metric of space-time is given by a tensor operator, $\widehat{g}_{\mu \nu}$, and the quantum vacuum 
gives rise to a stress-energy tensor operator, $\widehat{T}_{\mu}^{\nu}$, the vacuum expectation of the latter being zero, that is eq.(6) holds true. Our aim will be to get the vacuum expectation of the metric tensor operator in terms of the two-point correlations

$$
\left\langle\operatorname{vac}\left|\widehat{T}_{\mu}^{\lambda}(x) \widehat{T}_{\nu}^{\sigma}(y)\right| v a c\right\rangle .
$$

In the toy model I assume that quantum vacuum fluctuations possess spherical symmetry, that is they depend only on the radial coordinate, $r$, and the time, $t$. Thus we may use standard (or curvature) coordinates so that the (quantized) metric is

$$
d \widehat{s}^{2}=\widehat{A}(r, t) d r^{2}+\widehat{I r}^{2}\left(d \theta^{2}+\sin ^{2} \theta d \phi^{2}\right)-\widehat{B}(r, t) d t^{2} .
$$

where $\widehat{I}$ is the identity operator. In what follows I shall use units $c=1$, but write explicitly Newton's constant, $G$, for the sake of clarity.

The next task will be to get the operators $\widehat{A}(r, t)$ and $\widehat{B}(r, t)$ from the stress-energy tensor operator $\widehat{T}_{\mu \lambda}(r, t)$. As a guide I shall start from relations valid in classical gravity for a space-time of spherical symmetry in standard coordinates. It is a fortunate fact that these relations do not involve explicitly the time coordinate. The relations are 12

$$
\begin{aligned}
& A(r, t)=\left(1-\frac{2 G m(r, t)}{r}\right)^{-1}, m(r, t) \equiv 4 \pi \int_{0}^{r} \rho(x, t) x^{2} d x, \\
& B(r, t)=\exp \left[2 G \int_{0}^{r} \frac{m(x, t)+4 \pi x^{3} p(x, t)}{x^{2}-2 G m(x, t) x} d x\right]
\end{aligned}
$$

where $\rho \equiv T_{t}^{t}$ is the mass density and $p \equiv-T_{r}^{r}$ the radial pressure. In our model the transverse pressure, $q \equiv-T_{\theta}^{\theta}=-T_{\phi}^{\phi}$, may be different from the radial pressure, but it plays no role in the following. In quantized gravity the operators $\widehat{\rho}(r, t) \equiv \widehat{T_{t}^{t}}(r, t)$ and $\widehat{p}(r, t) \equiv-\widehat{T_{t}^{t}}(r, t)$ at different places may not commute. Therefore getting the quantized counterparts of eqs.(13) is not trivial. The problem is easier if we work to second order in Newton's constant, $G$, which is enough for the purposes of this paper. Thus I will write the first eqs.(13) in the form

$$
A(r, t)=1+\frac{2 G m}{r}+\frac{4 G^{2} m^{2}}{r^{2}}+O\left(G^{3}\right),
$$


Similarly the second eq.(13) may be written

$$
\begin{aligned}
B(r, t)= & 1+2 G \int_{0}^{r}\left[x^{-2} m(x)+4 \pi x p(x)\right] d x \\
& +4 G^{2} \int_{0}^{r}\left[x^{-3} m(x)^{2}+4 \pi m(x) p(x)\right] d x \\
& +2 G^{2}\left[\int_{0}^{r}\left[x^{-2} m(x)+4 \pi x p(x)\right] d x\right]^{2}+O\left(G^{3}\right) .
\end{aligned}
$$

Now I propose that the quantized counterparts of these equations are obtained with the replacements $\rho(r, t) \rightarrow \widehat{\rho}(r, t), p(r, t) \rightarrow \widehat{p}(r, t)$ plus the substitution of symmetrically ordered products of operators for the usual products of the corresponding classical quantities. Thus for instance

$$
p\left(r_{1}, t_{1}\right) p\left(r_{2}, t_{2}\right) \rightarrow \frac{1}{2}\left(\widehat{p}\left(r_{1}, t_{1}\right) \widehat{p}\left(r_{2}, t_{2}\right)+\widehat{p}\left(r_{2}, t_{2}\right) \widehat{p}\left(r_{1}, t_{1}\right)\right) .
$$

After that it is straightforward to get the desired vacuum expectations, that is

$$
\begin{aligned}
\langle v a c|\widehat{A}(r, t)| v a c\rangle & \simeq 1+\left\langle v a c\left|\frac{2 G}{r} \widehat{m}(r)+\frac{4 G^{2}}{r^{2}} \widehat{m}(r)^{2}\right| v a c\right\rangle \\
\widehat{m}(r) \equiv & \int_{0}^{r} \widehat{\rho}(x) 4 \pi x^{2} d x, \\
\langle v a c|\widehat{B}(r, t)| v a c\rangle \simeq & 1+\frac{2 G}{r}\left\langle v a c\left|\int_{0}^{r} x^{-2} \widehat{m}(x) d x+\int_{0}^{r} 4 \pi x \widehat{p}(x) d x\right| v a c\right\rangle \\
& +2 G^{2}\left\langle v a c\left|\left[\int_{0}^{r} x^{-2} \widehat{m}(x) d x+\int_{0}^{r} 4 \pi x \widehat{p}(x) d x\right]^{2}\right| v a c\right\rangle \\
& +2 G^{2}\left\langle v a c\left|\int_{0}^{r} 4 \pi[\widehat{m}(x) \widehat{p}(x)+\widehat{p}(x) \widehat{m}(x)] d x\right| v a c\right\rangle \\
& +2 G^{2}\left\langle v a c\left|2 \int_{0}^{r} x^{-3} \widehat{m}(x)^{2} d x\right| v a c\right\rangle .
\end{aligned}
$$

It may be realized that in these expressions the quantum operators $\widehat{\rho}$ and $\widehat{p}$ appear always in symmetrical order. The dependence on time of the operators $\widehat{\rho}$ and $\widehat{p}$ has not been explicitly shown, and it will not be written in 
what follows. It is understood that all quantities are defined at the same coordinate time with the metric (12). In eqs.(16) and (17) the terms of order $G$ cancel, taking eq. (6) into account. It is straightforward to write the terms of order $G^{2}$ in terms of the correlations of the stress-energy tensor that is

$$
\begin{aligned}
\frac{1}{2}\left\langle v a c\left|\widehat{\rho}\left(r_{1}\right) \widehat{\rho}\left(r_{2}\right)+\widehat{\rho}\left(r_{2}\right) \widehat{\rho}\left(r_{1}\right)\right| v a c\right\rangle & \equiv C_{\rho \rho}\left(r_{1}, r_{2}\right), \\
\frac{1}{2}\left\langle v a c\left|\widehat{p}\left(r_{1}\right) \widehat{p}\left(r_{2}\right)+\widehat{p}\left(r_{2}\right) \widehat{p}\left(r_{1}\right)\right| v a c\right\rangle & \equiv C_{p p}\left(r_{1}, r_{2}\right), \\
\frac{1}{2}\left\langle v a c\left|\widehat{\rho}\left(r_{1}\right) \widehat{p}\left(r_{2}\right)+\widehat{p}\left(r_{2}\right) \widehat{\rho}\left(r_{1}\right)\right| v a c\right\rangle & \equiv C_{\rho p}\left(r_{1}, r_{2}\right),
\end{aligned}
$$

but I shall not do that explicitly.

In a semiclassical description the vacuum expectations of the metric tensor operator should be taken as a classical metric tensor. That tensor is the same which would be produced by some classical stress-energy tensor which may be obtained by comparison of eq.(14) with eq.(16) and eq.(15) with eq.(17). For instance the former gives

$$
m_{c l}(r)=\frac{2 G}{r}\left\langle v a c\left|\widehat{m}(r)^{2}\right| v a c\right\rangle .
$$

Hence, taking into account the relations between $m_{c l}$ and $\rho_{c l}$ and between $\widehat{m}$ and $\widehat{\rho}$, we get

$$
\rho_{c l}(r)=8 \pi G\left[\frac{2}{r} \int_{0}^{r} x^{2} C_{\rho \rho}(r, x) d x-\frac{1}{r^{4}} \int_{0}^{r} x^{2} d x \int_{0}^{r} y^{2} d y C_{\rho \rho}(x, y)\right] .
$$

A similar, but more involved, expression may be obtained for $p_{c l}(r)$, but I shall not write it. The interesting result is that the (ficticious) classical density, $\rho_{c l}$, is the product of Newton constant $G$ times some average of the correlation between quantum fluctuations of the density. This makes plausible our proposals eqs.(9) to (11).

In summary, the arguments of the present paper strongly suggest that quantum vacuum fluctuations necessarily give rise to a curvature of spacetime which roughly agrees with that attributed to dark energy. However a rigorous derivation would be required, which is not possible in the absence of a quantum gravity theory. 


\section{References}

[1] Varun Sahni, Dark matter and Dark energy, Lect. Notes Phys. 653, 141-180 (2004). e-print Archive astro-ph/0403324v3.

[2] G. Hinshaw et al., Astrophys. J. Suppl. Ser. 180, 225-245 (2009); e-print Archive astro-ph/0803.0732 (2008).

[3] E. J. Copeland, M. Sami and S. Tsujikawa, Int. J. Mod. Phys. D 15, 1753 (2006); e-print Archive hep-th/0603057 (2006).

[4] T. Jacobson, Phys. Rev. Lett. 75, 1260 (1995); C. Eling, R. Guedens and T. Jacobson, Phys. Rev. Lett. 96, 121301 (2006).

[5] S. Weinberg, Rev. Mod. Phys. 61, 1 (1989).

[6] M. Novello and R. P. Neves, Class. Quant. Grav. 20, L67 (2003); M. Novello, Int. J. Modern Phys. D, 13, 1405 (2004); J. P. Gazeau and M. Novello, ArXiv:gr-qc/0610054v1 (2006).

[7] Zeldovich, Sov. Phys. Usp. 24, 216 (1981).

[8] T. Padmanabhan, Class. Quant. Grav. 22, L107 (2005).

[9] R. M. Wald, Quantum field theory in curved spacetimes and black hole thermodynamics. The University of Chicago Press, Chicago, 1994.

[10] S. Nojiri and S. D. Odintsov, Int. J. Geom. Meth. Mod. Phys. 4,115 (2007); e-print Archive hep-th/0807.0685.

[11] T. P. Sotiriou and V. Faraoni, e-print Archive gr-qc/0805.1726v3.

[12] J. L. Synge, Relativity. The general theory. Norht-Holland Pub. Co., Amsterdam, 1965. Page 273. 